\title{
Coronavirus - New Variants and New Symptoms
}

\author{
Raena Simon ${ }^{1}$, Abhishek Poojary², Ajay Rao H.T. ${ }^{3}$, Sharan S. Sargod ${ }^{4}$, Reshma Suvarna ${ }^{5}$, \\ Afreen Shabbir6, Nanditha Hegde ${ }^{7}$, Shrivya Saloni Mahaveeran ${ }^{8}$
}

1, 2, 3, 4, 5, 6, 7, 8 Department of Pediatric and Preventive Dentistry, Yenepoya Dental College, Mangalore, Karnataka, India.

\section{ABSTRACT}

Corona virus disease 2019, also known as Covid-19, is a public health crisis that is threatening the world. It is rapidly spreading and highly contagious. The etiological agent is SARS-CoV-2 (severe acute respiratory syndrome corona virus - 2). It is even more contagious than SARS - CoV and Middle East respiratory syndrome corona virus (MERS - CoV). This ailment was declared as a 'pandemic' by WHO and Public Health Emergency of International Concern (PHEIC). It was reported first in Wuhan, China in 2019. The most probable route of human to human transmission is mainly through respiratory droplets, touching or coming into contact with infected person or contaminated surfaces. Recently corona virus has mutated and new strains have been produced.

Among the new variants, the UK variant which is known as 'B.1.1.7' is 40 to 70 percent more transmissible than the original corona strain. New strains of corona virus have been producing new strange symptoms. These symptoms are called 'Non classic symptoms'. New strains are causing more weakness and lethargy. Oral manifestations are more common in the new variants. Pediatric symptoms vary depending on the stage of the disease, prior health status, and the presence of comorbidities.

For the first 3 months during Covid-19 pandemic, it was expected to be an immunizing non - relapsing disease. But many secondary infection cases are reported recently. On considering the modes of transmission, dentists are in a very high risk category, because of the close contact with patients and the nature of the dental treatment. This article mainly emphasizes the importance of new strains of corona virus and its symptoms, oral manifestations, pediatric symptoms, reinfection potential and dental considerations.

\section{KEY WORDS}

Covid-19, Variants, Coronavirus, Oral Manifestations,Dentistry.
Corresponding Author: Dr. Ajay Rao H. T., Yenepoya Dental College Deralaktte, Mangalore - 575018,

Karnataka, India.

E-mail: drajayrao@gmail.com

DOI: $10.14260 / \mathrm{jemds} / 2021 / 643$

How to Cite This Article:

Simon $R$, Poojary A, Rao HTA, et al. Coronavirus - new variants and new symptoms. J Evolution Med Dent Sci 2021;10(36):3166-3170, 10.14260/jemds/2021/643

Submission 20-01-2021, Peer Review 11-08-2021, Acceptance 18-08-2021, Published 06-09-2021.

Copyright @ 2021 Raena Simon et al. This is an open access article distributed under Creative Commons Attribution License [Attribution 4.0 International (CC BY 4.0)] 


\section{BACKGROUND}

The corona virus disease 2019, also called as Covid-19 is a newly discovered viral infection. It is rapidly spreading and highly contagious. ${ }^{1}$ Coronaviruses are non-segmented, positive sense, enveloped, RNA viruses which belong to the coronaviridae family. It was first discovered in Wuhan, China in 2019, later caused the outbreak of pneumonia in the rest of the world. ${ }^{2}$ The WHO and the 'Public Health Emergency of International Concern' (PHEIC) have declared Covid-19 as a 'pandemic'. ${ }^{2}$ As a treatment method, containment measures were implemented in China \& South Korea and this intervention has drastically reduced the incidence of new cases. Italy was the second country to obtain a prominent position in reporting new cases. Due to this emerging pandemic and its outbreak in the Italian population, the Italian government \& European Union promoted the measures of "flatten the curve" of Covid-19 infection. ${ }^{2}$

\section{Prevalence and Variants}

The etiological agent of Covid-19 is SARS-CoV-2(Severe acute respiratory syndrome corona virus - 2). This is different and more contagious than SARS-COV and Middle East respiratory syndrome corona virus (MERS-COV). ${ }^{1}$ The host receptor is similar to SARS-COV, which is Human angiotensin - converting enzyme - 2 (ACE - 2). According to data collected from the epicentre of the contagion in Italy, 29,692 SARS-CoV-2 deaths were recorded on May 14, 2020.3 The median age of death was 80 years and median age of infection was 62 years. ${ }^{3}$ Death was mainly due to co-morbidities wherein $68.1 \%$ had hypertension, $30.5 \%$ had diabetes Type 2 \& others. Acute respiratory distress syndrome was seen in majority (98.8\%) of the patients. ${ }^{3}$ Recently corona virus has mutated and new strains have been produced. The different variants are U.K variant (B.1.1.7), South African variant (B.1.351), Brazilian variant (P.1), California variant (CAL.20C) and Nigerian variant (B1525). Apart from these, 'N440K' and 'E484Q' variants of SARS - CoV - 2 have been detected in Maharashtra, Kerala and Telangana. ${ }^{4}$ In the first wave of Covid-19, adults were more infected than children, but in the past 2 to 3 months in U. S., there has been a significant increase in the percentage of children being diagnosed with Covid-19.5 Among the variants, the UK variant known as 'B.1.1.7', was first detected in September 2020. The studies show that it is 40 to 70 percent more transmissible than the original corona strain. Dr. Greg Poland, Professor of medicine and infectious diseases, reported that the U. K. variant is doubling every 10 days in the US, according to a new study. ${ }^{6}$ In India, the number of people who have tested positive for the UK variant has climbed to $150 . .^{7}$ The government has asked the states to fast-track contact tracing of these positive cases, and isolate and test the symptomatic contacts so as to curb the spread of infection that is known to be at least $70 \%$ more infectious than the original virus. ${ }^{8}$ The South African variant is recently detected in India and is more transmissible than UK variant. It affects the younger population more. ${ }^{9}$ According to recent reports, Brazilian and South African strains are detected in India which are more contagious and spread fast and vaccination can be less effective as scientists have told, although there is no proof. The South African and Brazilian variants easily infect a person's lungs than the UK variant. ${ }^{10}$

\section{Route of Transmission}

The main route of transmission of SARS-CoV-2 is usually through a symptomatic patient, but asymptomatic patients should be equally taken into consideration. ${ }^{3}$ Common route of human to human transmission is mainly through respiratory droplets, touching or coming into contact with infected person or contaminated surfaces. ${ }^{1}$ The reproductive volume is considered between 2 and 3.5, which is considered as a very high rate. One person could spread the infection from two to three other people, despite having no or mild symptoms. ${ }^{3}$ Since possibility of transmission is via the droplets, more attention has to be given to the eyes, because of the hypothesis that the virus could enter the tears through droplets and reach the nose \& respiratory tract via the nasolacrimal duct. ${ }^{11}$

\section{NEW SYMPTOMS}

As we know, new strange symptoms of new strains of corona virus have been reported. The new Covid-19 strains have reportedly given rise to seven new symptoms, apart from the usual symptoms. These include conjunctivitis, diarrhoea, discolouration of fingers or toes, aches and pains, sore throat, skin rashes and headache. ${ }^{4}$ At Spain's Hospital Universitario La Paz, according to a study conducted in Madrid, warning signs can be detected on tongue, palms and soles of the patient as skin rashes. ${ }^{12}$ These are called as 'Non Classic symptoms'. New strains are causing weakness and lethargy.

A rare, strange symptom of 'Covid-19' is 'Covid Tongue'. It may involve swelling and painful rash, or indentations on the sides of the tongue. An epidemiologist, Professor Tim Spector at King's college of London, had reported about an unusual sign in the mouth. ${ }^{13} \mathrm{He}$ also added about patients with a white or yellow "furry coating" on their tongues. More research is needed to confirm whether "COVID Tongue" could be a reliable litmus test for a corona virus infection. It is a harmless condition which resolves by itself within a few days or weeks, even though initially it appeared as a serious one. Apart from COVID tongue, around $15 \%$ of the patients complained of experiencing redness and burning sensation on their palms and soles of their feet. Some patients had scaly rashes on their feet and swollen toes looked as frostbitten. This condition is nicknamed as 'COVID toes'. It is a rare symptom and more and more cases of swelling on the fingers, toes and skin are being reported across the world. ${ }^{14}$ According to the lead researcher of the study, Dr. Almudena Nuno - Gon zalez, said in an interview, "Almost half of the patients with mild to moderate Covid-19 admitted in field hospital showed mucocutaneous findings." $15 \mathrm{He}$ also added, that special examination was needed from time to time for the oral cavity as it is also a cause of concern. Oral health is an important and vital part of overall health, and the ADA is continuing to examine the connection between the two as it relates to Covid-19. In London, new symptoms were found and it was published as a study by the Imperial College London, which include chills, loss of appetite, headache and muscle aches, related to the novel corona virus. There was some variation in symptoms depending on age, but chills were associated with Covid-19 across all age groups, which was reported by the study released by the REACT (Real - time Assessment of Community Transmission) team at the Imperial College London. ${ }^{16}$ The sudden appearance of rashes or raised bumps on the skin, which can last for hours can also 
be a sign of Covid-19 in the early days, another presentation of skin - related Covid-19 symptoms.

They can linger on for longer period and sometimes, even stay on for weeks even after one has been cured of the infection. The latest study has found that skin rashes have become a strong early indicator of corona virus infection amongst adults, which was later published in the British Journal of Dermatology. A normal rash can be considered as a sign of allergy, however, a rash accompanied with redness and a bump must be diagnosed as it can be due to the inflammation caused by Covid-19. Blood clotting is a side-effect which is increasingly reported by Covid-19 patients. It has been established that the SARS-CoV-2 virus can rapidly spread throughout the body, clot the blood vessels and disrupt healthy blood flow through the veins. Blood clotting can also affect young and healthy patients. These blood clots can strike unexpectedly, choke blood vessels and disturb the functioning of vital organs including the heart, kidneys, liver, which can be detrimental. Respiratory symptoms are not only the indicators, they are the classic markers of Covid-19 as well. One such devastating impact can be felt on core digestion and gastrointestinal functioning. Gastrointestinal complaints can be common with Covid-19. As per statistics, $53 \%$ of patients with Covid-19 go on to develop at least one gastrointestinal symptom during the course of their illness. These symptoms can be present at any early stage. Some people with Covid-19 infection complain of loss of appetite during the early stage, which is now been characterized as a warning sign of the infection. As Covid-19 disrupts your digestion, experiencing abdominal pain and cramps can commonly happen. In fact, in a lot of cases, patients admit to experience a stabbing abdominal pain during the course of infection. Nausea and vomiting are now being considered as signs of infection present in the early days. Another unusual side-effect or strange symptom of a Covid-19 infection can be weight fluctuation and metabolic loss. It is important to eat foods which help the body to heal, recover and build back the stamina during the time of infection. Hydration is also another important factor that should be kept in mind. ${ }^{17}$

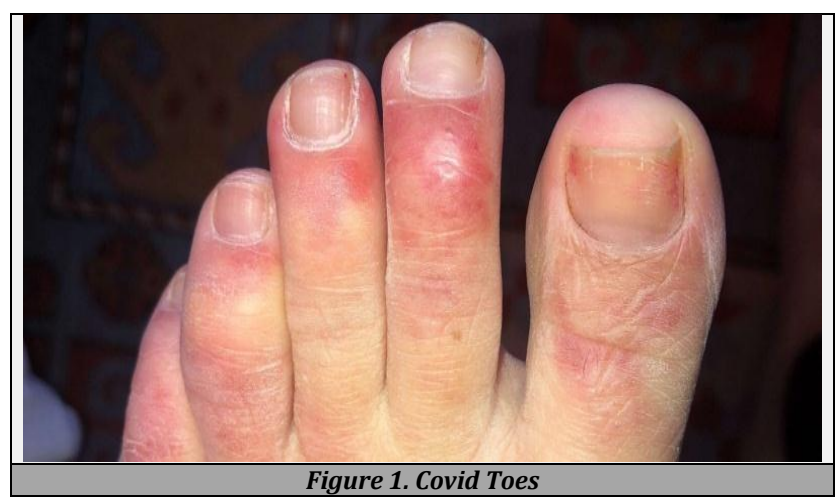

\section{Pediatric Considerations}

Pediatric symptoms depending on the stage of the disease, prior health status, and the presence of co-morbidities may vary a lot. According to the UK's National Health Services (NHS), main symptoms of Covid-19 are almost similar in children as that of adults. It includes new or continuous cough and a loss or change in their sense of smell and taste. Apart from these, some have symptoms of a cold such as a sore throat, congestion, or a runny nose and chills. One of the significant symptoms recorded in children was Multisystem inflammatory syndrome (MIS - C), which causes severe inflammation of various organs of the body, including heart, lungs, blood vessels, kidney digestive system, brain, skin or eyes. A COVID positive child who develops MIS - C shows various symptoms, ranging from skin rashes, high temperature and abdominal pain to diarrhoea and shortness of breath. All patients with MIS - $\mathrm{C}$ are not expected to have the same signs and symptoms and clinical features. This may include fatigue, rash, cardiac abnormalities, oropharyngeal erythema and dilation of conjunctival blood vessels. Covid-19 is rare and asymptomatic SARS - CoV - 2 is more frequent in children than in adults.

\section{Oral Manifestations}

Oral manifestations of Covid-19, as discussed above are the white patches and ulcers in the tongue, also called as Covid tongue. Epithelial cells of oral mucosa have high expression of ACE2 receptors. These cells may provide possible route for entry of coronavirus -19 , which indicate that oral cavity may be a potential site for risk of infection. ${ }^{18}$ Salivary glands may serve as an alternative route of infection. ${ }^{19}$ Other manifestations are dry mouth (46.3\%), diminished taste sensation (47.2\%). Dysguesia may be the first reported oral manifestation. ${ }^{20}$ Oral findings in children are similar to that of adults. As Martin et al. in 2020 presented case series with ulcers and blisters in patients who were affected with the disease. ${ }^{21}$ Treatment is similar to that of other oral lesions (hyluronic acid and chlorhexidine).

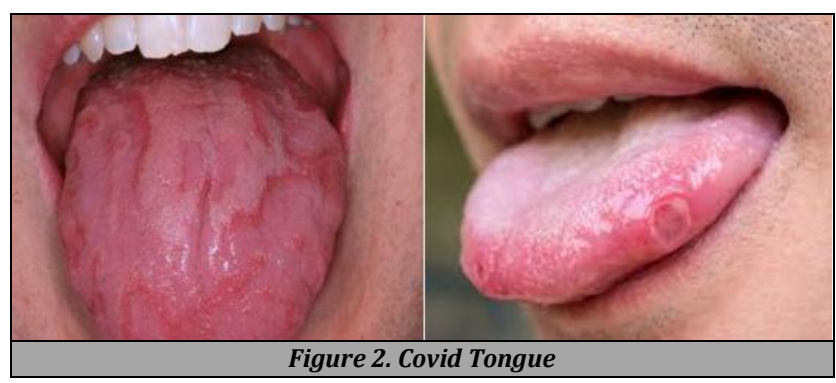

\section{Chances of Reinfection}

For the first 3 months of Covid-19 pandemic, it was expected to be an immunizing non-relapsing disease. There is a reported national case series of 11 virologically - confirmed Covid-19 patients those experienced a second clinically - and virologically - confirmed acute Covid-19 episode. According to the clinical history, they have discussed either re - infection or reactivation hypothesis. Larger studies including further virological, immunological and epidemiologic data are required to understand the mechanisms of these recurrences. ${ }^{22}$ Reinfection with Covid-19 is rare, as only a handful of cases are reported among the 42 million cases worldwide. The reinfection susceptibility of previously infected patients is not well understood. In Hong Kong, The Netherlands, Belgium, Ecuador, Israel and Australia, cases of Covid-19 reinfection have been reported. The first case of reinfection was recently reported in a patient in US, Reno, Nevada. ${ }^{23}$ The human body has two types of immunity which are innate and acquired immunity. The antibodies, IgM, 
normally appear within one to two weeks of time when any viral infection occurs. These antibodies mobilize the virus and then start to disappear slowly. A few weeks later after the infection has cleared, IgG antibodies appear and the levels of IgG antibody persist for many years, especially in case of certain childhood viruses such as varicella. However, this is not the case with coronavirus infections. Similar to other viral causes of upper respiratory tract infections, corona viruses have been known to cause reinfections. It appears that corona viruses ensure that the body's long term response is not that powerful against the virus. As per the previous studies of MERS and SARS-CoV infections, they have shown that neutralizing antibodies and total binding reduces slowly over 1 to 3 years. The previously infected persons will have limited or no ability to protect themselves from reinfection. The studies have also proven that patients with severe illness and prolonged viral shedding had higher antibody titres present for a longer duration of time.23

\section{Dental Considerations}

On considering the modes of transmission, the close contact with patients and the nature of the dental treatment, dentists are considered in a very high-risk category. The dental clinic could be a riskier environment to spread the virus. Asymptomatic patients infected with Covid-19 are of a great threat to dentists and other members of the dental team. Dentists, thereby, should be able to control and manage its spread and entertain a high level of awareness and integrity to deal with the disease. ${ }^{1}$

Since the oral cavity is a high-risk route for Covid-19 infection, the use of preoperative antimicrobial mouth rinse is recommended. ${ }^{24}$ For the dentists and the dental staffs, there are practical guidelines recommended by the Center for Disease Control and Prevention (CDC), the American Dental Association (ADA), and the World Health Organization, to have a control over the spread of Covid-19.

Similar to other contagious infections, these recommendations include personal protective equipment, hand washing, detailed patient evaluation, rubber dam isolation, antiretraction handpiece, mouth rinsing before dental procedures, and disinfection of the clinic. Mouth rinses incorporated with $0.2-1 \%$ povidone, instead of $0.05-0.1 \%$ of cetylpyridinium chloride or $1 \%$ hydrogen peroxide are usually recommended although there is no evidence of proven efficacy.

A particular point should be made about the efficacy of chlorhexidine mouth rinses against SARS-CoV-2, which is inefficient against the virus. On the other hand, a specific formula, including cyclodestrins combined with citrox, to enhance the delivery system and the efficacy against the virus could lower the viral load and reduce the nasopharyngeal microbiota.

Another mouth rinse that seems to be effective against the virus is represented by cetylpiridinuim chloride, and its use is proved as an antiviral agent through the destruction of the viral capsid. ${ }^{3}$ In addition to this, few guidelines and reports have provided useful information about the signs and symptoms of the disease, ways of transmission, and referral mechanisms to increase dentists' knowledge and prevention practices, so at a population level, they could contribute for disease control and prevention.

\section{Post Covid Syndrome}

Long COVID, as called as post COVID syndrome, which is a scary phenomenon. ${ }^{25}$ It is also known as a host of symptoms which mimics the classic symptoms like persisting cough, myalgia, recurring fever, breathlessness, delusion and in many cases, acute cardiac, respiratory and neurological manifestations. Scientists have a new name for the postsickness malaise and fatigue which affects Covid-19 patients through the course of their infection, which is post-acute sequelae of SARS-CoV-2 infection or PASC. Patients with Covid-19 who experienced more than five symptoms in their first week of illness were more likely to be a "Covid-19 long hauler," which researchers proved as having symptoms for longer than 28 days, according to a study published in the peer - reviewed journal called "Nature Medicine". ${ }^{26}$ It can cause lingering symptoms for patients who find it hard to make a healthy recovery. ${ }^{14}$ As most of the kids are asymptomatic and said to recover faster, doctors and parents can miss out on these symptoms or lingering side-effects. As studies show, most of the post COVID symptoms were detected in children who were symptomatic. Most common symptoms observed were insomnia and mental problems, respiratory disturbances, tiredness and chronic pain, inability to concentrate and confusion, risk of seizures and paralysis. ${ }^{25} \mathrm{~A}$ new research has suggested that the Covid shots may actually relieve symptoms in long Covid sufferers. According to a study conducted by a team of scientists from the University of Bristol, Covid vaccination may ease the symptoms in patients suffering from long term implications of Covid-19.27

Covid-19 has taken a heavy toll on our physical health as well as our mental health and wellbeing. During the fear driven times, we cannot help but wonder what if cough, cold or fever are signs of COVID or not. Scientists had coined a name, 'Coronaphobia' that was specifically related to corona triggered anxiety. Coronaphobia is a type of fear, which is defined as an excessive triggered response of the fear of virus causing Covid-19, leading to over concern about physiological symptoms, significant stress about personal and occupational loss, increased reassurance, and avoidance of public places and situations, which can cause marked impairment in daily life functioning. According to a study published in the Asian Journal of Psychiatry in Dec 2020, experts have detected three reigning characteristics of anxiety arising from Covid-19. The following are the associated symptoms -

1. Never ending worries leading to heart palpitations, loss of appetite and dizziness,

2. Constant overthinking which triggers fear and worry,

3. A sense of fear for attending public gatherings and events. According to a recent report published in online journal, symptoms of insomnia, anxiety and depression were more common in women than in men. Dr. Lily Brown, PhD, researcher in her report said, younger population are experiencing an increase in anxiety due to the virus as well as the pandemic created by it. The Centre for Disease Control and Prevention (CDC) has suggested various ways to tackle these problems of anxiety and stress. It encourages to take care of one's physical health and also to socialize with others. Besides that, cognitive behavioral therapy (CBT) has shown excellent 
results in treating anxiety efficiently. With the emergence of vaccine, the anxiety may have lowered down a bit , but the fear and phobia still haunts us. ${ }^{28}$

\section{CONCLUSIONS}

As we are aware about the fact that Covid vaccination has started all over the world, we must be as careful as we were at the beginning of this pandemic. Researchers have been saying, that the vaccine is effective against the new strains of coronavirus as well. As the new symptoms are related to oral cavity, importance of oral health must be enhanced among the general population. As dentists, we should do the preliminary oral examination thoroughly to rule out any of these symptoms. We must take proper precautions and take care of ourselves following the protocols. Always remember, prevention is better than cure.

Financial or other competing interests: None.

Disclosure forms provided by the authors are available with the full text of this article at jemds.com.

\section{REFERENCES}

[1] Khader Y, Al Nsour M, Al-Batayneh O, et al. Dentists' awareness, perception and attitude regarding COVID-19 and infection control: cross-sectional study among jordanian dentists. JMIR Public Health Surveill 2020;6(2):e18798.

[2] Spagnuolo G, De Vito D, Rengo S, et al. COVID-19 outbreak: an overview on dentistry. Int J Environ Res Public Health 2020;17(6):2094.

[3] D'Agostino S, D'Agostino L, Dolci M. Protection measures, dental education and Covid-19 spread: a brief narrative review. J Int Soc Prev Community Dent 2021;11(1):1-5.

[4] Symptoms of new COVID-19 strains: how are they different from old variant? Zee News 2021.

[5] Salazar JC. What are the new COVID-19 strains and do they infect children more easily? Connecticut Children's 2021.

[6] Frellick M. UK COVID-19 variant doubling every 10 days in the US: study. Medscape Medical News 2021.

[7] 150 people infected with UK variant of COVID19 in India: Government 2021

[8] Kaul R. UK Covid variant: 13 more test positive in India, total now at 71. Hindustan Times 2021.

[9] Explained: is discovery of South African strain of COVID19 a matter of concern for India? Indian Express 2021.
[10] UK, South Africa, Brazilian Covid strain cases in India now at 213. All India 2021.

[11] Qing H, Li Z, Yang Z, et al. The possibility of COVID-19 transmission from eye to nose. Acta Ophthalmol 2020;98(3):e388.

[12] Covid new strain symptoms: four unusual symptoms reported in COVID-19 patients. https://www.express.co.uk/

[13] Coronavirus: COVID tongue has been identified as a new coronavirus symptom, as per experts. Times of India 2021.

[14] Coronavirus: 5 new, unusual COVID symptoms which have just been discovered. Times of India 2021.

[15] Waugh R. Flu vaccine shot protects children against symptoms of coronavirus. Yahoo News 2021.

[16] New symptoms of COVID 19-chills, loss of appetite and muscle aches cites UK study. IANS 2021.

[17] Coronavirus symptoms: signs COVID-19 has impacted your digestion. Times of India 2021.

[18] Xu H, Zhong L, Deng J, et al. High expression of ACE2 receptor of 2019-nCoV on the epithelial cells of oral mucosa. Int J Oral Sci 2020;12(1):1-5.

[19] Xu J, Li Y, Gan F, et al. Salivary glands: potential reservoirs for COVID-19 asymptomatic infection. J Dent Res 2020;99(8):989.

[20] Chen L, Zhao J, Peng J, et al. Detection of 2019-nCoV in saliva and characterization of oral symptoms in COVID-19 patients. Cell Prolif 2020;53(12):e12923.

[21] Koticha PB, Pradhan D, Katge F, et al. CovID-19 in children: its impact on oral health and paediatric dentistry. International Journal of Science \& Healthcare Research 2020;5(3):377-89.

[22] Gousseff M, Penot P, Gallay L, et al. Clinical recurrences of COVID-19 symptoms after recovery: viral relapse, reinfection or inflammatory rebound? J Infec 2020;81(5):816-46.

[23] Selvaraj V, Herman K, Dapaah-Afriyie K. Severe, symptomatic reinfection in a patient with COVID-19. R I Med J (2013) 2020;103(10):24-6.

[24] Bescos R, Casas-Agustench P, Belfield L, et al. Coronavirus disease 2019 (COVID-19): emerging and future challenges for dental and oral medicine. J Dent Res 2020;99(9):1113.

[25] Long COVID risk in kids: what are the symptoms you should look for? Times of India 2021.

[26] Rodrguez A. These symptoms and risk factors may predict whether you could become a COVID-19 long hauler, study suggests. USA Today 2021.

[27] Coronavirus vaccine: COVID vaccines can relieve long COVID symptoms, claims research. Times of India 2021.

[28] What is Coronaphobia? Everything you need to know. Times of India 2021. 